\title{
Silhouette Scores for Arbitrary Defined Groups in Gene Expression Data and Insights into Differential Expression Results
}

\author{
Shitao Zhao, Jianqiang Sun, Kentaro Shimizu and Koji Kadota* ${ }^{*}$
}

\begin{abstract}
Background: Hierarchical Sample clustering (HSC) is widely performed to examine associations within expression data obtained from microarrays and RNA sequencing (RNA-seq). Researchers have investigated the HSC results with several possible criteria for grouping (e.g., sex, age, and disease types). However, the evaluation of arbitrary defined groups still counts in subjective visual inspection.

Results: To objectively evaluate the degree of separation between groups of interest in the HSC dendrogram, we propose to use Silhouette scores. Silhouettes was originally developed as a graphical aid for the validation of data clusters. It provides a measure of how well a sample is classified when it was assigned to a cluster by according to both the tightness of the clusters and the separation between them. It ranges from 1.0 to -1.0 , and a larger value for the average silhouette (AS) over all samples to be analyzed indicates a higher degree of cluster separation. The basic idea to use an AS is to replace the term cluster by group when calculating the scores. We investigated the validity of this score using simulated and real data designed for differential expression (DE) analysis. We found that larger (or smaller) AS values agreed well with both higher (or lower) degrees of separation between different groups and higher percentages of differentially expressed genes $\left(P_{\mathrm{DEG}}\right)$. We also found that the AS values were generally independent on the number of replicates $\left(N_{\text {rep }}\right)$. Although the $P_{\mathrm{DEG}}$ values depended on $N_{\text {rep }}$, we confirmed that both $\mathrm{AS}$ and $P_{\mathrm{DEG}}$ values were close to zero when samples in the data showed an intermingled nature between the groups in the HSC dendrogram.
\end{abstract}

Conclusion: Silhouettes is useful for exploring data with predefined group labels. It would help provide both an objective evaluation of HSC dendrograms and insights into the DE results with regard to the compared groups.

Keywords: Hierarchical sample clustering, Bioinformatics, Differential expression analysis, Silhouettes

\section{Background}

High-throughput technologies, including microarrays and RNA-seq, are widely used to monitor genome-wide expression levels in samples of interest and to compare expression patterns in different groups or conditions (e.g., healthy vs. tumor tissue samples) [1-6]. The latter, comparative analyses are often termed differential expression (DE) analyses and the identification of differentially expressed genes or transcripts (DEGs) is a common approach in studies of the molecular basis of traits $[7,8]$. RNA-seq is now the main method used to obtain expression data, but microarrays

\footnotetext{
* Correspondence: kadota@bi.a.u-tokyo.ac.jp

Graduate School of Agricultural and Life Sciences, The University of Tokyo, 1-1-1 Yayoi, Bunkyo-ku, Tokyo 113-8657, Japan
}

have provided important insights (e.g., [9]). A main difference between the two technologies is the nature of the expression data: microarrays yield continuous signal intensities, while RNA-seq data provides discrete counts $[10,11]$. To appropriately manipulate these expression data, several specialized models (e.g., the negative binomial (NB) model for RNA-seq count data [12-18]) have been proposed.

Another common approach for expression analyses is sample clustering (SC) based on similarity in expression patterns [19-21]. Utilizing its unsupervised characteristic, SC has been used to (i) detect previously unrecognized subtypes of cancer [22, 23], (ii) detect outliers (i.e., outlying samples) [24], (iii) represent overall similarities in expression among various organs [25, 26], and (iv) perform sanity 
checks to verify expected clustering patterns [27]. When using this approach, researchers can investigate SC results with several possible criteria for grouping (e.g., sex, age, and disease types). However, the evaluation of arbitrary defined groups still counts in subjective visual inspection. Numerical scores indicating the degree of separation between predefined groups would help in the objective assessment of the $\mathrm{SC}$ results.

Some researchers empirically know that an SC result of data designed for DE analysis (say, "DE data") roughly corresponds to the DE result when the groups for the $\mathrm{DE}$ analysis are evaluated with respect to the SC result [8]. If individual groups form distinct sub-clusters, where each sub-cluster consists only of members (or samples) in the particular group, DE analysis using such distinct groups would result in many DEGs. Conversely, if members (or samples) in each sub-cluster originate from multiple groups, no or few DEGs would be expected. However, objective evaluation of the relationship between SC results on DE data and the percentages of DEGs $\left(P_{\mathrm{DEG}}\right)$ remains lacking [8].

Silhouettes is a graphical aid for the interpretation and validation of cluster analysis [28]. In SC, silhouettes provide a measure of how well a sample is classified when it is assigned to a cluster according to both the tightness of the clusters and the separation between them. Therefore, the silhouette scores are calculated for individual samples. By taking the mean over all samples, the average silhouette (AS) value can be obtained. It ranges from 1.0 to - 1.0: a higher (or lower) AS value indicates higher (or lower) degree of separation between clusters. Silhouettes has been successfully used after clustering as a cluster validity measure [20, 29-31].

In this paper, we propose to use Silhouette for the objective evaluation of gene expression data based on arbitrary grouping criteria. Although they are independent of SC, silhouette scores measuring the degrees of separation between groups of interest would enable a more objective discussion about the SC result in terms of the groups. We here focus on single-factor DE data where only one grouping criterion is primarily of interest in relation to the DE result. We evaluated the relationship among SC results, DE results, and AS values, using both simulated and real expression data (RNA-seq and microarrays). We found silhouettes (i.e., AS values) to provide a relevant measure for the degrees of separation between groups of interest in SC results. We also found a positive correlation between AS values and DE results.

\section{Results}

In DE analyses, a gene expression matrix is typically generated, where each row indicates the gene (or derivatives), each column indicates the sample, and each cell indicates (i) counts for RNA-seq data or (ii) the signal intensity for microarray data. Our previous observation of the positive correlation between SC and DE results [8] was obtained from an RNA-seq dataset (referred to as Blekhman, for short) consisting of 20,689 genes $\times 18$ samples $(=3$ species $\times 2$ sexes $\times 3$ biological replicates (BRs)) [32]. The analysis was performed using a hierarchical SC (HSC) algorithm and a DE pipeline, both of which are provided in the R/Bioconductor package TCC [33-35]. TCC implements a robust normalization strategy (called DEGES [36]) that uses functions provided in four widely used packages (baySeq [37], edgeR [38, 39], DESeq [40], and DESeq2) [15]. For simplicity and/or the algorithmic advantage [41, 42], we only used TCC for the DE analysis of RNAseq data. Specifically, we used the default DE pipeline (iDEGES/edgeR-edgeR in [33] and EEE-E in [8]). When performing HSC for all input data, we used the clustering function clusterSample with default options ("1 Spearman's correlation coefficient $(r)^{\prime \prime}$ as a distance estimate and average-linkage agglomeration) in TCC.

Throughout this study, we filtered out genes with zero counts (or signals) in all samples. For HSC analyses, an additional filtering was performed where genes having identical expression patterns were collapsed. Expression data having those unique expression patterns were used for calculating distance defined as "1 - Spearman's $r$." This filtering procedure was intended to reduce the negative impact of genes with low expression levels when calculating the distance between samples. For example, the Blekhman data yielded 17,886 genes after the zero-count filtering and $\mathrm{DE}$ analyses were performed. After unique filtering, 16,560 genes were obtained, and HSC was performed using these genes. For simplicity, we focus on twogroup comparisons with three replicates for each group, i.e., (A1, A2, A3) vs. (B1, B2, B3), in most cases. In this study, we use the terms samples and replicates interchangeably. Our primary interest was to investigate the applicability of Silhouette for the objective evaluation of gene expression data based on arbitrary grouping criteria. By using silhouettes (i.e., AS values) as a relevant measure for the group differentiation in the HSC results, we reevaluated our previous observations (i.e., the positive correlation between HSC and DE results) [8].

\section{Representative Relationship between HSC and DE Results with AS}

We first demonstrate the relationship between HSC and DE results using a representative dataset, the Blekhman data obtained for three species (i.e., the three-group data): humans (HS), chimpanzees (PT), and rhesus macaques (RM) [32]. Briefly, Blekhman et al. studied expression levels in liver samples from three males (M1, M2, and M3) and three females (F1, F2, and F3) from each species/group. Figure 1a shows the HSC dendrogram based on a correlation distance $(1-r)$ metric and average-linkage 




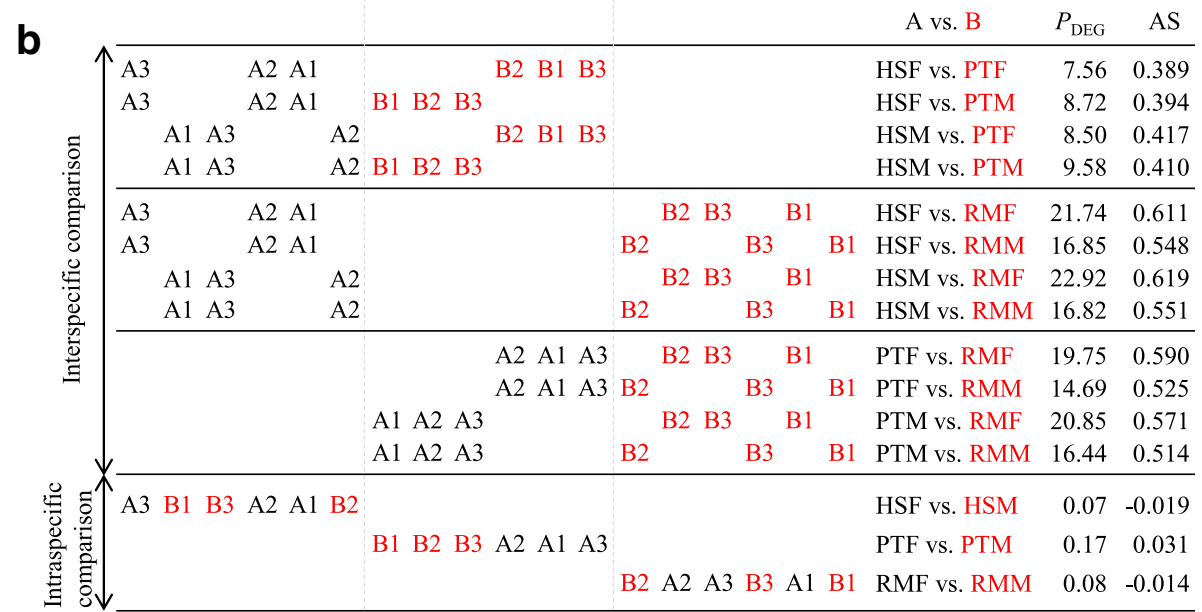

Fig. 1 Relationship between the shape of HSC and DE results. a HSC dendrogram for Blekhman data consisting of 16,560 genes $\times 18$ samples. The clustering was performed using the "clusterSample" function with default options in TCC. The unique filtering (from 17,886 genes to 16,560 genes with unique expression patterns across 18 samples) was internally performed in the function to reduce the negative effect on associations in low count regions when calculating Spearman's $r$ as a distance measure. $\mathbf{b}$ DE results from a total of 15 two-group comparisons with three replicates. The DE pipeline provided in TCC was applied to the Blekhman's count matrix consisting of 17,886 genes after zero-count filtering. The $P_{D E G}$ values and AS values for individual comparisons are provided on the right

agglomeration. There were three major clusters, each of which represented a particular species (HS, PT, and RM clusters) and the RM cluster was relatively distant from the other clusters. Different from the clear interspecific discrimination (i.e., high dissimilarity between species), we observed a very low degree of separation between sexes ( $\mathrm{F}$ vs. M) within each of the three major clusters. That is, samples labelled female (F) and male (M) were intermingled within each species, except for the PTF sub-cluster comprising three female samples (PTF1, PTF2, and PTF3).

Figure $1 \mathrm{~b}$ shows $15 \mathrm{DE}$ results for two-group comparisons. The percentages of DEGs $\left(P_{\mathrm{DEG}}\right)$ satisfying the $10 \%$ false discovery rate (FDR) threshold were obtained using TCC with default settings. The four $P_{\mathrm{DEG}}$ values for the HS vs. PT comparison (7.56-9.58\%) were much smaller than those for either the HS vs. RM (16.82-22.92\%) or the PT vs. RM comparison (14.69-20.85\%). These results are consistent with those of the original study [32] and can primarily be explained by the interspecific distances shown in Fig. 1a. Different from the interspecific comparisons, sex comparisons (F vs. M) showed extremely low $P_{\mathrm{DEG}}$ values $(0.07-0.17 \%)$. This is consistent with the lack of separation between female and male samples within each species in the HSC analysis (Fig. 1a).

Silhouette [28] has been successfully employed to estimate the appropriate number of clusters for gene expression data [20, 29-31]. Although Silhouette is generally used for the validation of clustering results, we here employ it independently from clustering. Technically, the term cluster is replaced with group in the silhouette calculation procedure. For each sample $i$, let $u_{i}$ be the average distance between $i$ and all other samples within the same group (e.g., group A). Let $v_{i}$ be the average distance between $i$ and the other group (e.g., group B), of which $i$ is not a sample member. The silhouette index $s_{i}$ for sample $i$ is 
calculated as $\left(v_{i}-u_{i}\right) / \max \left(u_{i}, v_{i}\right)$. The index $s_{i}$ ranges from -1 to 1 ; it is positive if $u_{i}<v_{i}$, zero if $u_{i}=v_{i}$, and negative if $u_{i}>v_{i}$. A larger $s_{i}$ value indicates increased group separation and vice versa. By taking the mean $s_{i}$ over all samples, the average silhouette (AS) value for each comparison can be obtained (Additional file 1a; right hand in Fig. 1b). The potential applicability of the silhouette unrelated to clustering has been described in the original study [28]. However, to the best of our knowledge, the current study is the first practical application of the concept to estimate the degree of separation between groups (not clusters) using gene expression data.

It is noteworthy that, in the eight RM-related inter-group comparisons, both $P_{\mathrm{DEG}}$ and AS values obtained from four RMF-related comparisons were consistently larger than those from the four RMM-related comparisons. For example, for the HSF vs. RMF comparison, $P_{\mathrm{DEG}}=21.74 \%$ and $\mathrm{AS}=0.611$, while for the HSF vs. RMM comparison, $P_{\mathrm{DEG}}=16.85 \%$ and $\mathrm{AS}=0.548$. This difference is primarily explained by the smaller average distance of samples in RMF (0.0475) than in RMM (0.0722). Small $P_{\text {DEG }}$ values $(0.07-0.17 \%)$ obtained for the sex (i.e., intragroup) comparisons can be explained by the similarity between inter-group distances and intra-group distances. In other words, two-group comparisons showing $\mathrm{AS} \approx 0$ would result in few, if any, DEGs. The numbers of DEGs (or $P_{\mathrm{DEG}}$ values) can, of course, vary with FDR thresholds and generally increase when the threshold is less restrictive.

Nevertheless, we confirmed that the general trends for the 15 two-group comparisons were the same at $1 \%, 5 \%$, $10 \%, 20 \%, 30 \%$, and 40\% FDR thresholds (Additional file $1 \mathrm{~b}$ ). Based on the definition of FDR, an increase in the $P_{\text {DEG }}$ value by loosening the FDR threshold does not necessarily indicate an increase in the true number of DEGs. For example, $P_{\mathrm{DEG}}=0.78 \%$ at a $40 \%$ FDR for the PTF vs. PTM comparison indicates that $0.78 \times 0.4=0.31 \%$ are non-DEGs, and the remaining $0.78 \times(1.0-0.4)=0.47 \%$ are, at least statistically, true DEGs. In our experience, the percentage of true DEGs (say $P_{\text {trueDEG }}$ ) generally approaches a constant value at a non-stringent FDR threshold, such as $30 \%$ or $40 \%$. In this case, the maximum $P_{\text {trueDEG }}$ value for any sex comparison was $\sim 0.5 \%$ (Additional file 1c). These results indicate that differences in $P_{\mathrm{DEG}}$ values with respect to the FDR threshold are not important.

Based on our visual evaluation, the AS values effectively represented the overall relationship between groups of interest in the HSC analysis (shown in Fig. 1a). We think the expressive power in cases of few or no DEGs in the dataset (i.e., $\mathrm{AS} \approx 0$ ) is practically promising, but increasing the correlation between $P_{\mathrm{DEG}}$ (or $P_{\text {trueDEG}}$ ) and AS is not practical. This is simply because the $P_{\mathrm{DEG}}$ value tends to increase as the number of replicates $\left(N_{\text {rep }}\right)$ increases [43], suggesting that the correlation is influenced by $N_{\text {rep. }}$.

\section{Effects of the Number of Replicates $\left(N_{\text {rep }}\right)$ on Parameter Estimates}

We next investigated the effects of $N_{\text {rep }}$ on $P_{\mathrm{DEG}}$ and AS values, using both simulated and real RNA-seq data. The simulated data were constructed as follows: twogroup comparison (A vs. B) with 40 replicates per group $\left(N_{\text {rep }}=40\right), 10,000$ total genes, of which $20 \%$ were DEGs (2000 DEGs and 8000 non-DEGs; $P_{\text {simDEG }}=20 \%$ ), the levels of DE were four-fold in individual groups, and the proportions of DEGs up-regulated in individual groups were the same (i.e., 1000 DEGs are up-regulated in group A). For a total of 80 samples (A1, A2, .., A40, B1, B2, ... B40), we obtained $P_{\mathrm{DEG}}=21.0 \%$ at a $10 \%$ FDR threshold, $\mathrm{AS}=0.2409$, and area under the ROC curve $(\mathrm{AUC})=$ 0.9986 . The AUC is a widely used measure of both the sensitivity and specificity of the DE pipeline $[7,8,33,36]$. The value (ranging from 0 to 1 ) can also be regarded as an overall indicator of the ability to distinguish true DEGs from non-DEGs. A larger AUC value indicates better DE separation and vice versa. The AUC value of 0.9986 indicates nearly perfect separation and the estimated $P_{\text {DEG }}$ value $(21.0 \%$ at $\mathrm{FDR}=0.1)$ is in good agreement with the true value (i.e., $20 \%$ DEGs or $P_{\text {simDEG }}=20 \%$ ).

The DE pipeline was used to examine subsets from the baseline matrix with 40 replicates per group $\left(N_{\text {rep }}=\right.$ 40). Bootstrap resampling was performed 100 times at $N_{\text {rep }}=3,6, \ldots$, and 30 (without replacement). Consistent the previous observations [43], the average $P_{\text {DEG }}$ values increased as a function of $N_{\text {rep }}$ (Fig. 2a). However, such an increasing trend was not observed for AS (Fig. 2b). This result indicates that the the silhouette (i.e., AS) is independent of $N_{\text {rep }}$. Note that the $P_{\mathrm{DEG}}$ value approached to the true value $\left(P_{\text {simDEG }}=20 \%\right)$ as $N_{\text {rep }}$ increased (Fig. $\left.2 \mathrm{a}\right)$. In general, the DE pipeline does not necessarily produce a well-ranked gene list in which true DEGs are top-ranked and non-DEGs are bottom ranked. Given the increase in AUC values in conjunction with increases in $P_{\text {DEG }}$ (Fig. 2c), this interpretation can be trusted in this case.

Next, the effects of $N_{\text {rep }}$ under different $P_{\text {simDEG }}$ conditions $\left(P_{\text {simDEG }}=10 \%, 5 \%, 2 \%, 1 \%, 0.5 \%, 0.1 \%\right.$, and $\left.0.02 \%\right)$ were investigated. We confirmed that $P_{\mathrm{DEG}}$, but not on AS, is dependent on $N_{\text {rep }}$ (Additional file 2). Different from the condition shown in Fig. $2\left(P_{\text {simDEG }}=20 \%\right)$, however, we observed a transition in the distribution of $P_{\mathrm{DEG}}$ values at around $P_{\text {simDEG }}=1 \%$. Although the $P_{\mathrm{DEG}}$ value monotonously increased as $N_{\text {rep }}$ increases when $P_{\text {simDEG }}$ was $20 \%$ or more, the $P_{\mathrm{DEG}}$ value switched to a monotonously decreasing trend when $P_{\text {simDEG }}$ was $0.1 \%$ or less. Overall, the $P_{\mathrm{DEG}}$ values approached the true values (i.e., the $P_{\text {simDEG }}$ values) as $N_{\text {rep }}$ increased. These results indicate that more accurate DE results can be obtained as $N_{\text {rep }}$ increases, irrespective of the true percentages of DEGs in the data. 


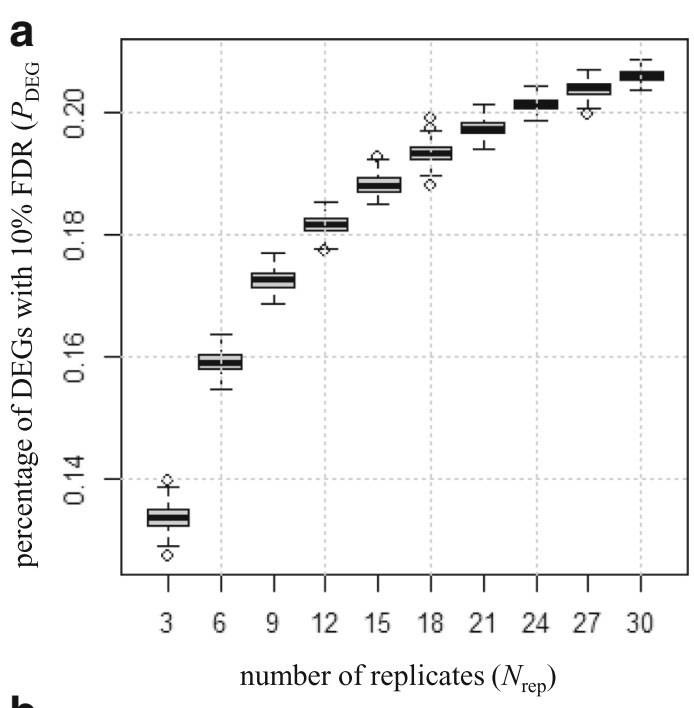

b
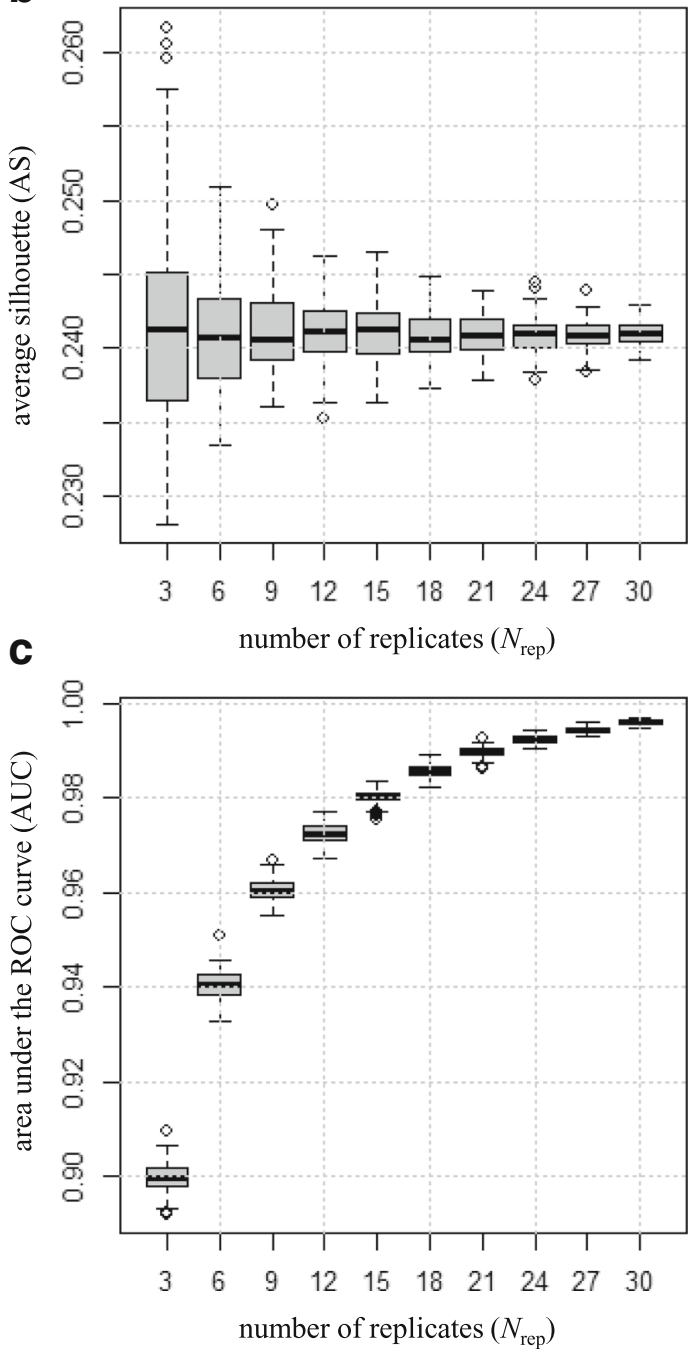

Fig. 2 Effects of $N_{\text {rep }}$ on parameter estimates (simulated data). Bootstrapping results (100 iterations) from simulated RNA-seq data consisting of 10,000 genes $\times 80$ samples with $P_{\text {simDEG }}=20 \%$ are shown. Vertical axes for the boxplots indicate: (a) $P_{\mathrm{DEG}}$ (b) $\mathrm{AS}$, and (c) AUC values. Horizontal axes indicate the $N_{\text {rep }}$ values $(3,6, \ldots, 30)$. It can be seen that $P_{\mathrm{DEG}}$ and $A U C$ values increase as a function of $N_{\text {rep, }}$ but AS values do not

A similar analysis was performed using another real RNA-seq dataset consisting of 7126 genes $\times 96$ samples $[43,44]$. Ten outlier samples were rejected, following the original study [43], and subsequent zero-count filtering of the original data yielded 6885 genes $\times 86$ samples (unique filtering did not have any effect for this dataset). For the data (called Schurch for short) comparing two groups (42 wild-type samples vs. $44 \Delta$ snf2 mutant samples), we obtained $P_{\mathrm{DEG}}=78.1 \%$ and $\mathrm{AS}=0.7289$. Note that the AUC value could not be calculated for the data because, different from simulated data, we do not know which genes are true DEGs. We investigated the effects of $N_{\text {rep }}$ on parameter estimates. The results were quite similar to those obtained using simulated data (shown in Fig. 2), i.e., $P_{\mathrm{DEG}}$ was dependent on $N_{\text {rep }}$, but AS was not (Additional file 3 ). Note that the distribution of $P_{\mathrm{DEG}}$ values obtained using TCC (Additional file 3a) was also similar to that obtained using edgeR [39] (Fig. 1a in [43]). This is quite reasonable because the DE pipeline implemented in TCC can be viewed as an iterative edgeR pipeline [8].

\section{Relationships between $P_{\mathrm{DEG}}$ and AS Values}

Next, we investigated the relationships between $P_{\text {DEG }}$ and AS values under a fixed $N_{\text {rep }}$ of 3 . Figure 3 shows the results for (a) Schurch, (b) simulated, and (c) the mixture. For simulated data, we examined $19 P_{\text {simDEG }}$ conditions from 5\% (black in Fig. 3b) to 0.95 (red in Fig. $3 \mathrm{~b})$. Overall, there was a strong positive correlation between $P_{\mathrm{DEG}}$ and AS values in this condition (Fig. 3c). However, the accurate estimation of $P_{\mathrm{DEG}}$ using AS is not realistic and accordingly is not a goal of the current study. This is mainly because $P_{\mathrm{DEG}}$ increases as a function of $N_{\text {rep }}$, while AS does not (Fig. 2; Additional file 3). In other words, the regression coefficients depend on $N_{\text {rep }}$. Most importantly, if one wants to calculate $P_{\mathrm{DEG}}$, there is no need to estimate the AS value; rather, it is only necessary to directly execute the DE pipeline. Nevertheless, as $P_{\text {DEG }}$ approaches 0 , AS also approaches 0 . This suggests that $P_{\mathrm{DEG}}$ values near 0 can be interpreted as a mathematical explanation for AS near 0, i.e., the samples in the two groups (A vs. B) were completely mixed. In statistical terms, this situation is essentially the same as the null hypothesis $\left(H_{0}: \mathrm{A}=\mathrm{B}\right)$. The acceptance of $H_{O}(\mathrm{AS}=0)$ indicates there are no or few DEGs in the two-group data $\left(P_{\mathrm{DEG}}=0\right)$. In this sense, AS could be used as 


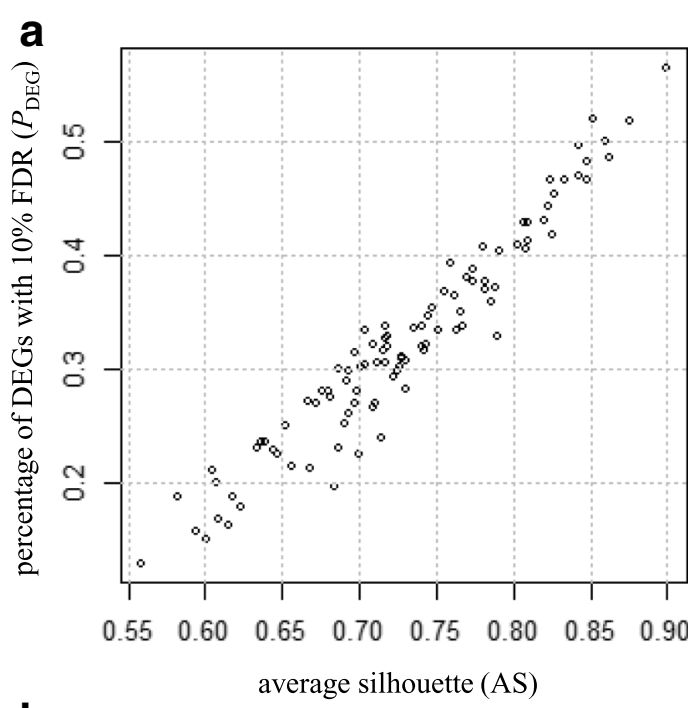

b
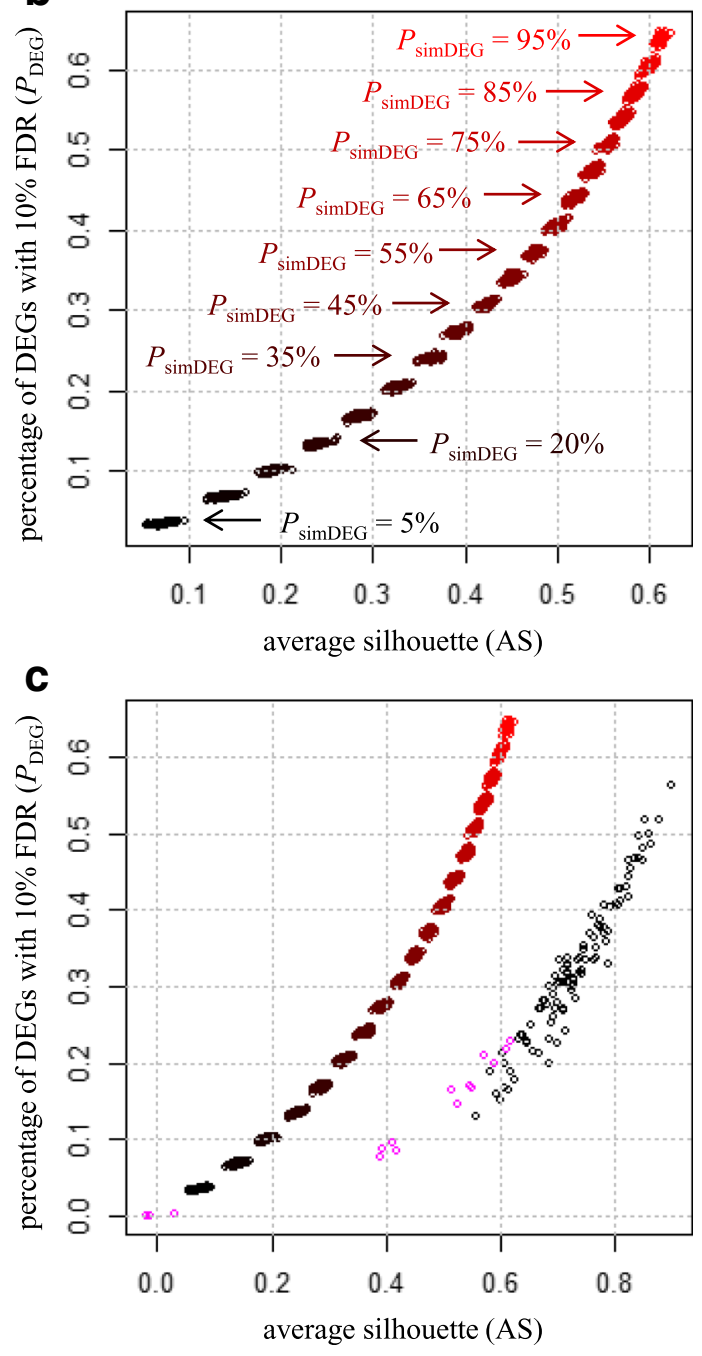

Fig. 3 Relationship between $P_{\mathrm{DEG}}$ and AS values. Scatter plots of $P_{\mathrm{DEG}} \mathrm{Vs}$. AS at $N_{\text {rep }}=3$ are shown. (a) Schurch data. The scatter plot shows a detailed relationship between $P_{\mathrm{DEG}}$ and AS values for Schurch data at $N_{\text {rep }}=3$ (Additional file $3 a$ and $3 b$ ). (b) simulated data under $P_{\text {simDEG }}=5 \%, \ldots, 95 \%$. The scatter plot for $P_{\text {simDEG }}=20 \%$ corresponds to the $P_{\mathrm{DEG}}$ (ranging from 0.1273 and 0.1397 ) and AS values (ranging from 0.2281 and 0.2617 ) for $N_{\text {rep }}=3$ shown in Fig. $2 b$. (c) the results for the mixture as well as the Blekhman data including 15 two-group comparisons shown in Fig. 1b (magenta)

helpful information for the interpretation of DE results, especially when only a few statistically significant DEGs are obtained.

It should be noted that the distribution shown in Fig. 3c (right panel) differs substantially from the distribution for real data (Blekhman [32] and Schurch [43]) and simulated data, but the shapes of the distributions were similar. For example, the $P_{\mathrm{DEG}}$ value at $\mathrm{AS}=0.6$ was approximately 0.6 for the simulated data, while $P_{\mathrm{DEG}}$ for real data was approximately 0.2 . Since the AS value for the simulated data at $P_{\mathrm{DEG}}=0.2$ was approximately 0.3 , the difference for AS at $P_{\mathrm{DEG}}=0.2$ was 0.3 . Similarly, the difference for $P_{\mathrm{DEG}}$ at $\mathrm{AS}=0.6$ was 0.4 . It should also be noted that the distribution of values for Blekhman (magenta) and Schurch (black with AS >0.5) was different (Fig. 3c). While low AS values $(-0.019-0.619)$ and low $P_{\mathrm{DEG}}$ values (0.07-22.92\%) were obtained for the Blekhman data, high AS values $(0.5585-0.8998)$ and high $P_{\text {DEG }}$ values $(13.03-$ $56.34 \%$ ) were obtained for the Schurch data. The difference can be explained by the intra-group distances. For the Schurch data, including 42 wild-type samples (group A) and $44 \Delta$ snf2 mutant samples (group B), the distances for groups A and B were 0.0144 and 0.0084 , respectively. The values obtained for the Schurch data were clearly smaller than those obtained for the Blekhman data (>0.04; Fig. 1a). According to a previous study [43], the Schurch data represents a best-case scenario for DE pipelines, since the within-group biological variation (BV) is low. As the BVs roughly correspond to the intra-group distances, many other real RNA-seq data may display low $P_{\mathrm{DEG}}$ and AS values compared to those obtained for the Schurch data.

\section{Analyses of two Additional Real RNA-Seq Datasets}

We further investigated two other real RNA-seq datasets available at the ReCount website [45]. The first dataset (called Bottomly [46]) consisted of 36,536 genes $\times 21$ samples. Briefly, Bottomly et al. studied the expression levels of two common inbred mouse strains used in neuroscience research, i.e., 10 C57BL/6J strains (A1, A2 ..., A10) and $11 \mathrm{DBA} / 2 \mathrm{~J}$ strains (B1, B2, ..., B11). DE analyses (i.e., estimates of $P_{\mathrm{DEG}}$ values) were performed using 13,932 genes after zero-count filtering. AS calculations and HSC were performed using 13,133 genes after unique filtering. 
The results for this dataset comparing 10 vs. 11 samples were $P_{\mathrm{DEG}}=11.0 \%$ at a $10 \%$ FDR threshold and $\mathrm{AS}=$ 0.1872. Regarding the effects of $N_{\text {rep, }}$, we observed similar trends to those obtained using the Schurch data (Fig. 3a), i.e., $P_{\mathrm{DEG}}$ increased as a function of $N_{\text {rep }}$, while AS did not (Additional file $4 \mathrm{a}-\mathrm{b}$ ). Despite similar trends, the values obtained for the Bottomly data were clearly lower than those for the Schurch data. For example, the Bottomly data (and Schurch data) showed on average $P_{\mathrm{DEG}}=$ $3.81 \%(32.4 \%)$ and $\mathrm{AS}=0.1874(0.7306)$ at $N_{\text {rep }}=3$ (Additional files 3 and 4). These findings suggested that the $P_{\mathrm{DEG}}$ values were lower for Bottomly than for Schurch because the AS value for Bottomly was lower than that of Schurch.

In general, high (or low) AS values indicate clear (or unclear) separation of groups. The HSC dendrogram for the Bottomly data showed relatively unclear separation of two groups (Additional file 4c; $\mathrm{AS}=0.1872$ ) compared to the separation of groups for the Schurch data (Additional file 3c; AS $=0.7289$ ). Nevertheless, as also implied by the positive AS value, the degree of inter-group separation for the Bottomly data was not random. For example, by visual inspection, we identified four clusters, each of which consisted only of samples within the same group (Additional file 4c). These clusters primarily explained the estimated $P_{\mathrm{DEG}}$ values and positive AS values. The highest values for $P_{\mathrm{DEG}}(=24.7 \%)$ and $\mathrm{AS}(=0.3524)$ among 100 trials at $N_{\text {rep }}=3$ were obtained when comparing (A3, A4, A6) vs. (B1, B3, B8). This is reasonable because five of the six samples (except for A3) were members of either the $A 2$ or $B 1$ cluster. Relatively high values can be obtained by comparing two groups in which all members belong to either the $A 2$ or $B 1$ clusters. Indeed, we observed $P_{\mathrm{DEG}}=34.8 \%$ and $\mathrm{AS}=0.4623$ when comparing (A2, A4, A6) vs. (B1, B2, $\mathrm{B} 3$ ), though this comparison was not included in the 100 original trials shown in Additional file 4a-b.

Different from the Schurch data where the impact of sampling effects shrunk as $N_{\text {rep }}$ increased (Additional file 3a), we did not observe shrinkage for the Bottomly data around $N_{\text {rep }}=3-7$ (Additional file 4a). This can also be explained by the four clusters mentioned above. For example, the highest values for $P_{\mathrm{DEG}}(=23.5 \%)$ and AS $(=0.2699)$ among 100 trials at $N_{\text {rep }}=6$ were obtained when comparing (A2, A3, A4, A6, A7, A9) vs. (B1, B2, B3, B4, B8, B10). All eight samples in the $A 2$ and $B 1$ clusters was included in the comparison. Additionally, a comparison between the two clusters, i.e., (A2, A4, A6, A7) vs. (B1, B2, B3, B8), yielded $P_{\mathrm{DEG}}=31.8 \%$ and $\mathrm{AS}=0.3701$. Accordingly, the decreases in $P_{\mathrm{DEG}}(=31.8 \%$ to $23.5 \%)$ and AS (0.3701 to 0.2699 ) values by the addition of four samples (A3, A9, $\mathrm{B} 4$, and B10) not included in the two clusters are reasonable. We observed that the impact of sampling effects tends to shrink as $N_{\text {rep }}(>7)$ increases. This is probably because the maximum number of samples in the four clusters is seven for the $B 4$ cluster; the addition of samples not included in the cluster can contribute to decreases in the $P_{\mathrm{DEG}}$ and $\mathrm{AS}$ values.

The second dataset (called Cheung [47]) consisted of 52,580 genes $\times 41$ samples. Briefly, Cheung et al. studied the expression levels of human B-cells using 17 females (A1, A2, ..., A17) and 24 males (B1, B2, ..., B24). The $\mathrm{DE}$ analyses (i.e., estimates of $P_{\mathrm{DEG}}$ values) were performed using 12,410 genes after zero-count filtering. AS calculation and HSC were performed using 11,738 genes after unique filtering. The results for this dataset comparing 17 vs. 24 samples were $P_{\mathrm{DEG}}=0.169 \%, \mathrm{SNR}=1.023$, and $\mathrm{AS}=0.0118$. The values were considerably lower than those obtained for both the Schurch and Bottomly data and were similar to those for the three sex comparisons (Fig. 1b). This result is intuitively reasonable, as gene expression levels in B-cells are not expected to differ greatly between females and males.

We did not observe an increasing trend for $P_{\mathrm{DEG}}$ values as $N_{\text {rep }}$ increased (Additional file 5a). The average $P_{\text {DEG }}$ values for 100 trials at $N_{\text {rep }}=3,5,7,9,11,13$, and 15 were $0.631 \%, 0.291 \%, 0.399 \%, 0.254 \%, 0.492 \%, 0.325 \%$, and $0.219 \%$, respectively. These values as well as the distribution were quite similar to those obtained from simulated data with $P_{\text {simDEG }}=0.5 \%$ (Page 5 in Additional file 2a). This result suggests that the increase of $N_{\text {rep }}$ does not contribute to the increase of $P_{\mathrm{DEG}}$ when AS is near 0 . Since AS is independent of $N_{\text {rep }}$, no or few DEGs $\left(P_{\mathrm{DEG}}<1 \%\right)$ would be obtained when AS $<0.1$ for count data (Additional file $5 \mathrm{~b}$ ). The intermingled nature of the HSC dendrogram for the Cheung data (Additional file 5c) also supports this inference; AS can be utilized to interpret the DE results.

\section{Analysis of two Microarray Datasets}

We finally investigated two microarray datasets obtained using the Affymetrix Rat Genome 230 2.0 Array (GPL1355). The first dataset (called Nakai [4]) consisted of 31,099 probesets (which can be viewed as genes) $\times 24$ samples $(=3$ tissues $\times 2$ conditions $\times 4$ BRs). Briefly, Nakai et al. studied the expression levels of genes in brown adipose tissues (BAT), white adipose tissues (WAT), and liver tissues (LIV). They compared two conditions (fed vs. fasted for $24 \mathrm{~h}$ ) for each tissue type. We here denoted the fed BAT samples BAT_fed, the $24 \mathrm{~h}$-fasted LIV samples $L I V_{-}$fas, and so on. To quantify expression from the probe-level data (i.e., Affymetrix CEL files), we applied three algorithms (MAS [48], RMA [49], and RobLoxBioC [50]). Different from RNA-seq data represented as integer counts, microarray data are expressed as continuous signals and in most cases are log-transformed. We therefore applied a specialized DE pipeline for microarray data provided in the package limma [51], instead of the DE pipeline used for RNA-seq data in TCC.

As expected based on the nature of microarray expression signals, zero signal values were not obtained for any 
genes in all samples and all genes displayed unique expression patterns. Accordingly, the subsequent analysis of microarray data was performed based on total set of genes $(=31,099)$. The HSC dendrogram for the Nakai data displayed three major clusters corresponding to the three tissue types (LIV, WAT, and BAT clusters) for all quantification algorithms (MAS, RMA, and RobLoxBioC; Additional file 6a). Since the experimental design and the HSC dendrogram were very similar to those of the Blekhman data (Fig. 1), these microarray data can be regarded as the counterpart.

We performed 15 two-group comparisons with four BRs for each group, i.e., (A1, A2, A3, A4) vs. (B1, B2, B3, B4). Overall, we observed highly similar trends for the Nakai data and the Blekhman data (Additional file 6b). For MAS-quantified data, for example, four $P_{\text {DEG }}$ values in the BAT vs. WAT comparison (24.49-34.98\%) were smaller than those in the BAT vs. LIV comparison (41.7944.63\%) or WAT vs. LIV comparison (39.74-44.05\%). Different from the clear inter-tissue differentiation (i.e., high dissimilarity between tissues), we detected a relatively low degree of separation between conditions (fed vs. fasted) within each of the three major clusters. The $P_{\mathrm{DEG}}$ values for the fed vs. fasted comparison were $4.5-8.79 \%$. Of these three comparisons, the intra-BAT comparison (i.e., BAT_fed vs. BAT_fas) showed the highest $P_{\mathrm{DEG}}(8.79 \%)$ and AS (0.207) values.

We observed similar results for RobLoxBioC-quantified data and relatively dissimilar results for RMA-quantified data. In particular, for the RMA-quantified data, we detected higher $P_{\mathrm{DEG}}$ and AS values compared to those of the other data. There are several potential explanations. RMA treats a batch of arrays simultaneously, while MAS and RobLoxBioC treat each array independently. RMA tends to overestimate sample similarity [52]. Combinations of DE pipelines with different quantification algorithms might also explain the higher $P_{\mathrm{DEG}}$ values observed in RMA-quantified data: limma is more compatible with MAS than RMA [53, 54]. Nevertheless, we observed a clear positive relationship between $P_{\mathrm{DEG}}$ and AS values, suggesting that AS is also applicable to microarray data.

The second dataset (called Kamei [55]) consisted of 31,099 genes $\times 10$ samples (five BRs per group). Briefly, Kamei et al. compared gene expression in livers for rats fed a low-iron diet (approximately $3 \mathrm{ppm}$ iron) for 3 days and a normal diet (48 ppm iron) as a control. The $P_{\text {DEG }}$ and AS values obtained (Iron_def vs. Control) were close to zero and the HSC dendrogram showed an intermingled structure (Additional file 7). These results indicate that the Kamei data can be regarded as a counterpart of the Cheung data (Additional file 5). AS can be utilized as supporting information to interpret DE results for both RNAseq and microarray data, especially when no or few DEGs were obtained.
We should note that one sample (Iron_def1) was a clear outlier in the HSC dendrogram for the RMA-quantified data, but not in the other dendrograms (Additional file 7). Iron_def3 was the most distant from the other samples in MAS- and RobLoxBioC-quantified data. This difference can also be explained by tendency of RMA to overestimate sample similarity [52]. Indeed, the average distance (0.007) among samples in RMA-quantified data was considerably lower than those for the other datasets ( 0.043 for MAS and 0.037 for RobLoxBioC). The expression levels for the two microarray datasets (Nakai and Kamei) were obtained using the same device (i.e., the Affymetrix Rat Genome 2302.0 Array), indicating that the datasets can be directly compared. The average distances among ten liver samples in the Kamei data were clearly lower than those among eight liver samples (LIV) in the Nakai data $(0.078$ for MAS, 0.022 for RMA, and 0.070 for RobLoxBioC). These results suggest that the differences in the most distant samples in the Kamei data (Iron_def1 in RMA data and Iron_def3 in the other data) are within the error range.

HSC dendrograms of the merged data provided several insights (Additional file 8). First, the ten liver samples in Kamei data formed a tight cluster, even after adding the Nakai data, and formed a larger cluster when the eight liver samples from the Nakai data were included, confirming the overall similarities among various tissues (i.e., a sanity check) [25-27]. Second, compared to 24-h fasting, the short-term iron-deficient diet might not result in significant differences in gene expression. This conclusion is supported by adding other publicly available dataset(s) for identical (or highly similar) tissues. It may be more important to add independent, publicly available datasets than to perform more detailed analyses using a single dataset. Third, an appropriate distance measure is important. The distance was defined here as ( 1 - Spearman's $r$ ); this definition is widely used $[21,27]$. Since the distance ranges from 0 to 2 , the interpretation is relatively easy compared to the interpretation of Euclidean distances, which range from 0 to $\infty$. We indeed understood the extremely high similarity among the ten liver samples in the Kamei data in the context of the very small distance values. In general, distance information is not interpreted so broadly in HSC analyses, but examinations of both the distance $(1-r)$ and AS may be useful.

\section{Discussion}

In this study, we proposed to use silhouettes (i.e., AS values) as an objective measure for the degrees of separation between groups of interest based on expression data. To our knowledge, the use of AS independent from HSC is the first practical application in the field of expression analysis. Our main findings are (i) AS is an effective indicator of the overall relationship in the HSC dendrogram based on arbitrary grouping criteria; (ii) AS values are 
independent of $N_{\text {rep }}$, while $P_{\mathrm{DEG}}$ values obtained from DE analysis are fundamentally dependent on $N_{\text {rep }}$; and (iii) there is a positive correlation between AS and $P_{\mathrm{DEG}}$ values under a fixed $N_{\text {rep. }}$. It is not necessary to estimate $P_{\text {DEG }}$ from AS values because DE results (including $P_{\mathrm{DEG}}$ ) can directly be obtained via the $\mathrm{DE}$ pipeline. The AS provides helpful information for interpreting DE results as well as HSC results.

Based on the current results, we conclude that our calculation procedure for AS is appropriate. The procedure consists of 1) filtering genes with low expression, 2) calculating distances among samples, and 3) calculating the AS values based on distance estimates. The high similarity among samples in the Kamei data could be detected by investigating the distances defined as ( 1 - Spearman's $r$ ). Considering this finding in addition to other data, some samples could be misidentified as outliers (e.g., Iron_def1 in Additional files 7 and 8). In addition to the AS value obtained for the groups of interest, (i) the investigations of distances among samples and/or groups in the dataset and (ii) comparison with other datasets obtained from the same or similar samples are practically important.

Of course, there are true outliers, e.g., ten outlying samples in the original Schurch data [43, 44]. We manually eliminated the ten outliers as determined in the original study [44] and analyzed 86 clean samples in this dataset (Fig. 3a; Additional file 3). The values obtained without outliers $\left(P_{\mathrm{DEG}}=78.1 \%\right.$ and $\left.\mathrm{AS}=0.7289\right)$ were clearly higher than those with outliers $\left(P_{\mathrm{DEG}}=74.7 \%\right.$ and AS $=0.6530$ ), indicating the importance of developing methods for the automatic detection of outliers $[55,56]$. Our preliminary analysis for the original data using an existing method [57] successfully detected nine of the ten true outliers as well as three false positives. We obtained a promising result $\left(P_{\mathrm{DEG}}=77.6 \%\right.$ and $\mathrm{AS}=$ $0.7301)$ using the remaining 84 samples. Rational removal of outlying samples would yield better DE results. We expect that AS would help objective evaluation of the changes in the DE results accompanying outlier removal.

In practice, Silhouettes can be utilized as supporting information to interpret DE results, especially when no or few DEGs are obtained. As demonstrated by several examples (e.g., Additional file 7), we actually encounter such expression data. Silhouettes enables us to discuss the DE results as well as SC dendrograms more objectively.

\section{Conclusion}

Silhouettes is useful for exploring data with predefined group labels. It would help provide both an objective evaluation of SC dendrograms and insights into the DE results with regard to the compared groups. The use of this measure would enable a more objective discussion about the SC result in terms of the groups.

\section{Methods}

Most of the analyses were performed using $\mathrm{R}$ (ver. 3.3.2) [34] and Bioconductor [35]. The versions of major $R$ packages used in the study were TCC ver. 1.14.0, edge $R$ ver. 3.16.5, ROC ver. 1.50.0, cluster ver. 2.0.5, affy ver. 1.44.0, and RobLoxBioC ver. 0.9. R-codes are provided in Additional file 10.

\section{Simulated Data}

The two-group simulated data were produced using the "simulateReadCounts" function in TCC [33]. The variance $(V)$ of the NB distribution can generally be modeled as $V=\mu+\phi \mu^{2}$. The empirical distribution of read counts to obtain the mean $(\mu)$ and dispersion $(\phi)$ parameters of the NB model was obtained from Arabidopsis data (three BRs for both treated and non-treated samples) in [58]. The output of the simulateReadCounts function is stored in the TCC class object with information about the simulated conditions and is therefore ready-to-analyze for both the DE analysis and HSC. These data were used to obtain Fig. 2, Fig. 3, and Additional file 2.

\section{Four RNA-Seq Data}

Blekhman's mammalian data were obtained from the supplementary website (http://genome.cshlp.org/content/ suppl/2009/12/16/gr.099226.109.DC1/suppTable1.xls) [32]. The raw count matrix consisting of 20,689 genes $\times 36$ samples $(=3$ species $\times 2$ sexes $\times 3$ BRs $\times 2$ technical replicates $)$ was collapsed by summing the data for technical replicates, giving a reduced number of columns in the matrix (i.e., 18 samples; 3 species $\times 2$ sexes $\times 3 \mathrm{BRs}$ ). These data were used to obtain Fig. 1, Fig. 3, Table 1, and Additional file 1.

Schurch's yeast data were obtained from the GitHub website (https:/github.com/bartongroup/profDGE48/tree/ master/Preprocessed_data) [43]. After merging the count vectors for a total of 96 samples, data from 10 outlying samples (WT_rep21, WT_rep22, WT_rep25, WT_rep28, WT_rep34, WT_rep36, Snf2_rep06, Snf2_rep13, Snf2_rep25, and Snf2_rep35) were eliminated. Subsequent data eliminations (named no_feature, ambiguous, too_low_aQual, not_aligned, and alignment_not_unique) yielded a count matrix consisting of 7126 genes $\times 86$ samples. These data were used to obtain Fig. 3 and Additional file 3.

Bottomly's mouse data were [46] obtained from the ReCount website (http://bowtie-bio.sourceforge.net/recount/ countTables/bottomly_count_table.txt) [45] and consisted of 36,536 genes $\times 21$ samples. These data were used to obtain Additional file 4.

Cheung's human data [47] were obtained from the ReCount website (http://bowtie-bio.sourceforge.net/recount/ countTables/cheung_count_table.txt) [45] and consisted of 
52,580 genes $\times 41$ samples. These data were used to obtain Additional file 5 .

\section{Two Rat Microarray Data}

Nakai's probe-level data (.CEL files) were obtained from the ArrayExpress website [59] through an $\mathrm{R}$ package ArrayExpress [60] by applying "GSE7623." The MASquantified data were obtained using the mas 5 function in the R/Bioconductor package affy [61]. Expression signals less than 1 were set to 1 and were subsequently $\log _{2}$ transformed. The RMA-quantified data were obtained using the rma function in the same package, i.e., affy. The output of the function was already $\log _{2}$-transformed. The RobLoxBioC-quantified data were obtained using the robloxbioc function in the $\mathrm{R}$ package RobLoxBioC [50]. The expression signals less than 1 were set to 1 and were subsequently $\log _{2}$-transformed. These data were used to obtain Additional files 6 and 8 .

Kamei's probe-level data (.CEL files) were obtained from the ArrayExpress website [59] using the R package ArrayExpress [60] by applying "GSE30533." The subsequent procedures were the same as those described for the Nakai data. These data were used to obtain Additional files 7 and 8 .

Note that the quantification procedure was performed using $\mathrm{R}$ ver. 3.1.3 (affy ver. 1.44.0) because we encountered an error when executing the functions mas5 and robloxbioc in $\mathrm{R}$ ver. 3.3.2 (affy ver. 1.52.0).

\section{HSC and DE Analyses}

The HSC was performed using the clusterSample function with default options (" 1 - Spearman's $r$ " as the distance and unique expression patterns as an objective low-count filtering method) in TCC [33]. The DE analysis was performed using three functions (calcNormFactors, estimateDE, and getResult) with default options which use functions in the package edge $R$ [39]. The genes were ranked in ascending order according to $p$-values. The ranks were used to calculate AUC values when analyzing simulated data (Fig. 2 and Additional file 2). The AUC values were calculated using the $A U C$ function in the package ROC. The $p$-values were adjusted for multiple-testing with the Benjamini-Hochberg procedure. The adjusted $p$-values (i.e., $q$-values) were used to obtain the numbers of DEGs satisfying an arbitrarily defined FDR threshold (mainly 10\%).

\section{Calculation of Average Silhouette (AS) Values}

The AS values were calculated using the silhouette function in the package cluster. Examples of the procedure to estimate AS values are given in Additional file 9.

\section{Additional files}

\begin{abstract}
Additional file 1: Detailed results for Blekhman's RNA-seq count data. (a) Silhouette indices $\left(s_{i}\right)$ for each sample $i$ and the average (AS). The sample names (A1, A2, A3, B1, B2, or B3) for $i$ correspond to those shown in Fig. $1 \mathrm{~b}$. (b) $P_{\mathrm{DEG}}$ values at various FDR thresholds (1\%,5\%,10\%, 20\%, 30\%, and $40 \%$ FDR). The values at 10\% FDR were the same as those shown in Fig. 1b. (c) Percentages of true DEGs $\left(P_{\text {trueDEG }}\right)$, defined as $P_{\text {DEG }} \times(1-$ FDR threshold $)$, at corresponding FDR thresholds shown in (b). (XLSX 19 kb)
\end{abstract}

Additional file 2: Effects of $N_{\text {rep }}$ on parameter estimates (simulated count data). Bootstrapping results for simulated data under different $P_{\text {simDEG }}$ values are shown: $P_{\text {simDEG }}=10 \%$ (Page 1), 5\% (Page 2), $2 \%$ (Page 3), $1 \%$ (Page 4), $0.5 \%$ (Page 5), 0.1\% (Page 6), and 0.02\% (Page 7). Other legends are the same as those in Fig. 2. (PPTX $110 \mathrm{~kb})$

Additional file 3: Results for Schurch's RNA-seq count data. For (ab), Bootstrapping results for Schurch data comparing 42 wild-type samples and $44 \Delta$ snf2 mutant samples are shown. Legends are the same as those in Fig. 2. (c) HSC dendrogram. Two distinct clusters, a wild-type cluster (right side) and $\Delta$ snf2 mutant cluster (left side), can be seen. The intra-group distances within 42 wild-type samples and $44 \Delta$ snf2 mutant samples were 0.0144 and 0.0084 , respectively. (d) Scatter plots of $P_{\mathrm{DEG}}$ vs. AS at $N_{\text {rep }}=3$ (black), 6 (blue), and 9 (sky blue). (PPTX $65 \mathrm{~kb}$ )

Additional file 4: Results for Bottomly's RNA-seq count data. For ( $a-b)$, Bootstrapping results for Bottomly data comparing $10 \mathrm{C} 57 \mathrm{BL} / 6 \mathrm{~J}$ strains $(A 1, A 2 \ldots, A 10)$ vs. 11 DBA/2 J strains (B1, B2, .., B11) are shown. (c) HSC dendrogram. For explanation, four clusters are defined in (d) the HSC dendrogram: the $B 1$ cluster (consisting of B1, B2, B3, and B8), A8 cluster $(A 8, A 9$, and $A 10)$, $A 2$ cluster (A2, A4, and A6), and B4 cluster (B4, B5, B6, B7, $B 9, B 10$, and B11). (d) Scatter plots of $P_{\mathrm{DEG}}$ Vs. AS at $N_{\text {rep }}=3$ (black), 6 (blue), and 9 (sky blue). (PPTX 55 kb)

Additional file 5: Results for Cheung's RNA-seq count data. For ( $a-b)$, Bootstrapping results for Cheung data comparing 17 females ( $A 1, A 2$, A17) vs. 24 males (B1, B2, ..., B24) are shown. (c) HSC dendrogram. (d) Scatter plots of $P_{\mathrm{DEG}}$ Vs. AS at $N_{\text {rep }}=3$ (black), 6 (blue), and 9 (sky blue). (PPTX $58 \mathrm{~kb}$ )

Additional file 6: Results for Nakai's microarray data. (a) HSC dendrogram for Nakai data consisting of 31,099 genes $\times 24$ samples and (b) $P_{\mathrm{DEG}}$ and AS values from a total of 15 two-group comparisons with $N_{\text {rep }}=4$ are shown: MAS-quantified data (Page 1), RMA-quantified data (Page 2), and RobLoxBioCquantified data (Page 3). (PPTX $76 \mathrm{~kb}$ )

Additional file 7: Results for Kamei's microarray data. HSC dendrograms for (a) MAS-, (b) RMA-, and (c) RobLoxBioC-quantified data are shown. These data consist of 31,099 genes $\times 10$ samples and compares two conditions (five Iron_def samples vs. five Contro/ samples). The $P_{\mathrm{DEG}}$ and AS values are also shown on the right side of the dendrogram. (PPTX $49 \mathrm{~kb}$ )

Additional file 8: HSC dendrograms for merged microarray data (Nakai + Kamei). HSC dendrograms for (a) MAS-, (b) RMA-, and (c) RobLoxBioCquantified data are shown. These data consist of 31,099 genes $\times 34$ samples (24 from Nakai and 10 from Kamei data). (PPTX 62 kb)

Additional file 9: Examples of AS estimates for two- and three-group data. The procedures for analyzing Nakai's MAS-quantified data consisting of 31,099 probesets $\times 24$ samples are provided. Example 1 compares three-group data with four BRs, 4 BAT_fed samples vs. 4 WAT_fed samples vs. 4 LIV_fed samples, with $A S=0.460$. Example 2 compares three-group data with two BRs, "BAT_fed 1 and 2" vs. "WAT_fed 1 and 2" vs. "LIV_fed1 and 2," with $A S=0.438$. Example 3 compares three-group data with two BRs, "BAT_fed 1 and BAT_fas1" vs. "BAT_fed2 and BAT_fas2" vs. "BAT_fed3 and BAT_fas3," with $A S=-0.185$. Example 4 compares two-group data with four BRs, 4 BAT_fed samples vs. 4 WAT_fed samples, with $A S=0.374$. Example 5 compares two-group data with four BRs, 4 BAT_fed samples vs. 4 LIV_fed samples, with $\mathrm{AS}=0.657$. (R $3 \mathrm{~kb})$

Additional file 10: $R$-codes for analyses. This zipped file includes a total of 23 R-code files. Results can be obtained by executing scripts in the order of the serial numbers $X X$ in the filename "rcode_ $X X \ldots . . . "$ Note that two files ("rcode_08_Add6_pre.R" and "rcode_10_Add7_pre.R") must be executed using $R$ ver. 3.1.3 (affy ver. 1.44.0) instead of $R$ ver. 3.3.2 (affy ver. 1.52.0). (ZIP 33 kb) 


\section{Abbreviations}

AS: Average silhouette; AUC: the area under the ROC curve; BAT: Brown adipose tissue; BR: Biological replicate; BV: Biological variation; DE: Differential expression; DEG: Differentially expressed gene; F: Female; FDR: False discovery rate; $H_{0}$ : null hypothesis; HS: Homo sapiens; HSC: Hierarchical sample clustering: LIV: Liver tissue; M: Male; NB: Negative binomial (distribution or model); $N_{\text {rep: }}$ Number of (biological) replicates; $P_{\text {DEG: }}$ Percentage of estimated DEGs (satisfying basically $10 \%$ FDR) by TCC; $P_{\text {simDEG: }}$ Percentage of DEGs when generating simulated data; $P_{\text {trueDEG: }}$ Percentage of true DEGs defined as $P_{\mathrm{DEG}} \times$ (1.0 - FDR threshold); PT: Pan troglodytes; RM: Rhesus macaques; ROC: Receiver operating characteristic; SC: Sample clustering; TCC: Tag Count Comparison; V: Variance; WAT: White adipose tissue

\section{Acknowledgments}

Not applicable.

\section{Funding}

This work was supported by JSPS KAKENHI Grant Number JP15K06919.

\section{Availability of Data and Materials}

Blekhman's mammalian data were obtained from the supplementary website (http://genome.cshlp.org/content/suppl/2009/12/16/gr.099226.109.DC1/ suppTable1.xls). Schurch's yeast data were obtained from the GitHub website (https://github.com/bartongroup/profDGE48/tree/master/Preprocessed_data). Bottomly's mouse data were obtained from the ReCount website (http:// bowtie-bio.sourceforge.net/recount/countTables/bottomly_count_table.txt). Cheung's human data were obtained from the ReCount website (http:// bowtie-bio.sourceforge.net/recount/countTables/cheung_count_table.txt) Nakai's probe-level data (.CEL files) were obtained from the ArrayExpress website through an R package ArrayExpress by applying "GSE7623." Kamei's probe-level data (.CEL files) were obtained using the $\mathrm{R}$ package ArrayExpress by applying "GSE30533." The R-codes for obtaining current results are provided in Additional file 10

\section{Authors' Contributions}

SZ performed the analysis and drafted the manuscript. JS maintained the package TCC and provided the critical comments. KS supervised the critical discussion and refined the paper. KK refined the paper, confirmed the analysis results, and led this project. All the authors read and approved the final manuscript.

\section{Ethics Approval and Consent to Participate}

Not applicable.

\section{Consent for Publication}

Not applicable.

\section{Competing Interests}

The authors declare that they have no competing interests.

\section{Publisher's Note}

Springer Nature remains neutral with regard to jurisdictional claims in published maps and institutional affiliations.

Received: 14 September 2017 Accepted: 12 January 2018 Published online: 01 March 2018

\section{References}

1. Miki R, Kadota K, Bono H, Mizuno Y, Tomaru Y, Carninci P, Itoh M, Shibata K, Kawai J, Konno H, Watanabe S, Sato K, Tokusumi Y, Kikuchi N, Ishii Y, Hamaguchi Y, Nishizuka I, Goto H, Nitanda H, Satomi S, Yoshiki A, Kusakabe M, DeRisi JL, Eisen MB, lyer VR, Brown PO, Muramatsu M, Shimada H, Okazaki Y, Hayashizaki Y. Delineating developmental and metabolic path ways in vivo by expression profiling using the RIKEN set of 18,816 fulllength enriched mouse cDNA arrays. Proc Natl Acad Sci U S A. 2001;98(5): 2199-204

2. Ichikawa Y, Ishikawa T, Takahashi S, Hamaguchi Y, Morita T, Nishizuka I, Yamaguchi S, Endo I, Ike H, Togo S, Oki S, Shimada H, Kadota K, Nakamura S, Goto H, Nitanda H, Satomi S, Sakai T, Narita I, Gejyo F, Tomaru Y, Shimizu K, Hayashizaki Y, Okazaki Y. Identification of genes regulating colorectal carcinogenesis by using the ADMS (algorithm for diagnosing malignant state) method. Biochem Biophys Res Commun. 2002;296(2):497-506.

3. Shimoji T, Kanda H, Kitagawa T, Kadota K, Asai R, Takahashi K, Kawaguchi N, Matsumoto S, Hayashizaki Y, Okazaki Y, Shinomiya K. Clinico-molecular study of dedifferentiation in well-differentiated liposarcoma. Biochem Biophys Res Commun. 2004;314(4):1133-40.

4. Nakai Y, Hashida H, Kadota K, Minami M, Shimizu K, Matsumoto I, Kato H, Abe K. Up-regulation of genes related to the ubiquitin-proteasome system in the brown adipose tissue of 24-h-fasted rats. Biosci Biotechnol Biochem. 2008;72(1):139-48.

5. Kawaoka S, Kadota K, Arai Y, Suzuki Y, Fujii T, Abe H, Yasukochi Y, Mita K, Sugano S, Shimizu K, Tomari Y, Shimada T, Katsuma S. The silkworm W chromosome is a source of female-enriched piRNAs. RNA. 2011;17(12):2144-51.

6. Lin Y, Golovnina K, Chen ZX, Lee HN, Negron YL, Sultana H, Oliver B, Harbison ST. Comparison of normalization and differential expression analyses using RNA-Seq data from 726 individual Drosophila Melanogaster. BMC Genomics. 2016;17:28.

7. Kadota K, Shimizu K. Evaluating methods for ranking differentially expressed genes applied to MicroArray quality control data. BMC Bioinformatics. 2011: 12:227.

8. Tang M, Sun J, Shimizu K, Kadota K. Evaluation of methods for differential expression analysis on multi-group RNA-seq count data. BMC Bioinformatics. 2015;16:361.

9. Bullard JH, Purdom E, Hansen KD, Dudoit S. Evaluation of statistical methods for normalization and differential expression in mRNA-Seq experiments. BMC Bioinformatics. 2010;11:94

10. Mortazavi A, Williams BA, McCue K, Schaeffer L, Wold B. Mapping and quantifying mammalian transcriptomes by RNA-Seq. Nat Methods. 2008;5(7):621-8.

11. Marioni JC, Mason CE, Mane SM, Stephens M, Gilad Y. RNA-seq: an assessment of technical reproducibility and comparison with gene expression arrays. Genome Res. 2008;18(9):1509-17.

12. Robinson MD, Smyth GK. Small-sample estimation of negative binomial dispersion, with applications to SAGE data. Biostatistics. 2008;9(2):321-32.

13. Anders S, McCarthy DJ, Chen Y, Okoniewski M, Smyth GK, Huber W Robinson MD. Count-based differential expression analysis of RNA sequencing data using R and Bioconductor. Nat Protoc. 2013:8(9):1765-86.

14. Zhou $X$, Lindsay $H$, Robinson MD. Robustly detecting differential expression in RNA sequencing data using observation weights. Nucleic Acids Res. 2014;42(11):e91.

15. Love Ml, Huber W, Anders S. Moderated estimation of fold change and dispersion for RNA-seq data with DESeq2. Genome Biol. 2014;15(12):550.

16. Ching T, Huang S, Garmire LX. Power analysis and sample size estimation for RNA-Seq differential expression. RNA. 2014;20(11):1684-96.

17. Sun X, Dalpiaz D, Wu D, S Liu J, Zhong W, Ma P. Statistical inference for time course RNA-Seq data using a negative binomial mixed-effect model. BMC Bioinformatics. 2016;17(1):324.

18. Hardcastle TJ. Generalized empirical Bayesian methods for discovery of differential data in high-throughput biology. Bioinformatics. 2016;32(2):195-202.

19. de Souto MC, Costa IG, de Araujo DS, Ludermir TB, Schliep A. Clustering cancer gene expression data: a comparative study. BMC Bioinformatics. 2008:9:497.

20. Jaskowiak PA, Campello RJ, Costa IG. On the selection of appropriate distances for gene expression data clustering. BMC Bioinformatics. 2014;15(Suppl 2):S2.

21. Reeb PD, Bramardi SJ, Steibel JP. Assessing dissimilarity measures for sample-based hierarchical clustering of RNA sequencing data using Plasmode datasets. PLoS One. 2015:10(7):e0132310.

22. Alizadeh AA, Eisen MB, Davis RE, Ma C, Lossos IS, Rosenwald A, Boldrick JC, Sabet H, Tran T, Yu X, Powell Jl, Yang L, Marti GE, Moore T, Hudson J Jr, Lu L, Lewis DB, Tibshirani R, Sherlock G, Chan WC, Greiner TC, Weisenburger DD, Armitage JO, Warnke R, Levy R, Wilson W, Grever MR, Byrd JC, Botstein D, Brown PO, Staudt LM: Distinct types of diffuse large B-cell lymphoma identified by gene expression profiling. Nature 2000, 403(6769):503-511.

23. Bittner $M$, Meltzer $P$, Chen $Y$, Jiang $Y$, Seftor $E$, Hendrix M, Radmacher $M$, Simon R, Yakhini Z, Ben-Dor A, Sampas N, Dougherty E, Wang E, Marincola F, Gooden C, Lueders J, Glatfelter A, Pollock P, Carpten J, Gillanders E, Leja D, Dietrich K, Beaudry C, Berens M, Alberts D, Sondak V. Molecular classification of cutaneous malignant melanoma by gene expression profiling. Nature. 2000;406(6795):536-40.

24. Alon U, Barkai N, Notterman DA, Gish K, Ybarra S, Mack D, Levine AJ. Broad patterns of gene expression revealed by clustering analysis of tumor and 
normal colon tissues probed by oligonucleotide arrays. Proc Natl Acad Sci U S A. 1999:96(12):6745-50.

25. Kadota K, Miki R, Bono H, Shimizu K, Okazaki Y, Hayashizaki Y. Preprocessing implementation for microarray (PRIM): an efficient method for processing cDNA microarray data. Physiol Genomics. 2001;4(3):183-8.

26. Qin Y, Pan J, Cai M, Yao L, Ji Z. Pattern genes suggest functional connectivity of organs. Sci Rep. 2016;6:26501.

27. Danielsson F, James T, Gomez-Cabrero D, Huss M. Assessing the consistency of public human tissue RNA-seq data sets. Brief Bioinform. 2015;16(6):941-9.

28. Rousseeuw PJ. Silhouettes: a graphical aid to the interpretation and validation of cluster analysis. J Comput Appl Math. 1987;20:53-65.

29. Gat-Viks I, Sharan R, Shamir R. Scoring clustering solutions by their biological relevance. Bioinformatics. 2003;19(18):2381-9.

30. Bandyopadhyay S, Mukhopadhyay A, Maulik U. An improved algorithm for clustering gene expression data. Bioinformatics. 2007;23(21):2859-65.

31. Lord E, Diallo AB, Makarenkov V. Classification of bioinformatics workflows using weighted versions of partitioning and hierarchical clustering algorithms. BMC Bioinformatics. 2015;16:68.

32. Blekhman R, Marioni JC, Zumbo P, Stephens M, Gilad Y. Sex-specific and lineage-specific alternative splicing in primates. Genome Res. 2010;20(2): 180-9.

33. Sun J, Nishiyama T, Shimizu K, Kadota K. TCC: an R package for comparing tag count data with robust normalization strategies. BMC Bioinformatics. 2013;14:219

34. R Core Team. R: A language and environment for statistical computing. Vienna: R Foundation for Statistical Computing; 2016. https://www.r-project.org/.

35. Gentleman RC, Carey VJ, Bates DM, Bolstad B, Dettling M, Dudoit S, Ellis B, Gautier L, Ge Y, Gentry J, Hornik K, Hothorn T, Huber W, lacus S, Irizarry R, Leisch F, Li C, Maechler M, Rossini AJ, Sawitzki G, Smith C, Smyth G, Tierney $L$, Yang JY, Zhang J. Bioconductor: open software development for computational biology and bioinformatics. Genome Biol. 2004:5:R80.

36. Kadota K, Nishiyama T, Shimizu K. A normalization strategy for comparing tag count data. Algorithms Mol Biol. 2012;7(1):5.

37. Hardcastle TJ, Kelly KA. baySeq: empirical Bayesian methods for identifying differential expression in sequence count data. BMC Bioinformatics. 2010;11:422

38. Robinson MD, Oshlack A. A scaling normalization method for differential expression analysis of RNA-seq data. Genome Biol. 2010;11:R25.

39. Robinson MD, McCarthy DJ, Smyth GK. edgeR: a Bioconductor package fo differential expression analysis of digital gene expression data. Bioinformatics. 2010;26(1):139-40.

40. Anders S, Huber W. Differential expression analysis for sequence count data. Genome Biol. 2010;11:R106.

41. Dillies MA, Rau A, Aubert J, Hennequet-Antier C, Jeanmougin M, Servant N, Keime C, Marot G, Castel D, Estelle J, Guernec G, Jagla B, Jouneau L, Laloë D, Le Gall C, Schaëffer B, Le Crom S, Guedj M, Jaffrézic F, French StatOmique Consortium. A comprehensive evaluation of normalization methods for Illumina high-throughput RNA sequencing data analysis. Brief Bioinform. 2013;14(6):671-83.

42. Maza E. In Papyro comparison of TMM (edgeR), RLE (DESeq2), and MRN normalization methods for a simple two-conditions-without-replicates RNASeq experimental design. Front Genet. 2016;7:164.

43. Schurch NJ, Schofield P, Gierliński M, Cole C, Sherstnev A, Singh V, Wrobel N, Gharbi K, Simpson GG, Owen-Hughes T, Blaxter M, Barton GJ. How many biological replicates are needed in an RNA-seq experiment and which differential expression tool should you use? RNA. 2016;22(6):839-51.

44. Gierliński M, Cole C, Schofield P, Schurch NJ, Sherstnev A, Singh V, Wrobel N, Gharbi K, Simpson G, Owen-Hughes T, Blaxter M, Barton GJ. Statistical models for RNA-seq data derived from a two-condition 48-replicate experiment. Bioinformatics. 2015:31(22):3625-30.

45. Frazee AC, Langmead B, Leek JT. ReCount: a multi-experiment resource of analysis-ready RNA-seq gene count datasets. BMC Bioinformatics. 2011:12:449.

46. Bottomly D, Walter NA, Hunter JE, Darakjian P, Kawane S, Buck KJ, Searles RP, Mooney M, McWeeney SK, Hitzemann R. Evaluating gene expression in C57BL/6J and DBA/2J mouse striatum using RNA-Seq and microarrays. PLoS One. 2011;6(3):e17820.

47. Cheung VG, Nayak RR, Wang IX, Elwyn S, Cousins SM, Morley M, Spielman RS. Polymorphic cis- and trans-regulation of human gene expression. PLoS Biol. 2010;8(9):e1000480.

48. Hubbell E, Liu WM, Mei R. Robust estimators for expression analysis. Bioinformatics. 2002;18(12):1585-92.
49. Irizarry RA, Hobbs B, Collin F, Beazer-Barclay YD, Antonellis KJ, Scherf U, Speed TP. Exploration, normalization, and summaries of high density oligonucleotide array probe level data. Biostatistics. 2003;4(2):249-64.

50. Kohl M, Deigner HP. Preprocessing of gene expression data by optimally robust estimators. BMC Bioinformatics. 2010;11:583.

51. Ritchie ME, Phipson B, Wu D, Hu Y, Law CW, Shi W, Smyth GK. Limma powers differential expression analyses for RNA-sequencing and microarray studies. Nucleic Acids Res. 2015;43(7):e47.

52. Giorgi FM, Bolger AM, Lohse M, Usadel B. Algorithm-driven artifacts in median polish summarization of microarray data. BMC Bioinformatics. 2010;11:553.

53. Kadota K, Nakai Y, Shimizu K. A weighted average difference method for detecting differentially expressed genes from microarray data. Algorithms Mol Biol. 2008;3:8.

54. Kadota K, Nakai Y, Shimizu K. Ranking differentially expressed genes from Affymetrix gene expression data: methods with reproducibility, sensitivity, and specificity. Algorithms Mol Biol. 2009;4:7.

55. Pimentel MA, Clifton DA, Clifton L, Tarassenko L. A review of novelty detection. Signal Process. 2014;99:215-49.

56. Kadota K, Tominaga D, Akiyama Y, Takahashi K. Detecting outlying samples in microarray data: a critical assessment of the effect of outliers on sample classification. Chem-Bio Informatics J. 2003:3(1):30-45.

57. Kadota K, Ye J, Nakai Y, Terada T, Shimizu K. ROKU: A novel method for identification of tissue-specific genes. BMC Bioinformatics. 2006;7:294.

58. Di Y, Schafer DW, Cumbie JS, Chang JH. The NBP negative binomial model for assessing differential gene expression from RNA-Seq. Stat Appl Genet Mol Biol. 2011;10:art24

59. Kolesnikov N, Hastings E, Keays M, Melnichuk O, Tang YA, Williams E, Dylag M, Kurbatova N, Brandizi M, Burdett T, Megy K, Pilicheva E, Rustici G, Tikhonov A, Parkinson H, Petryszak R, Sarkans U, Brazma A. ArrayExpress update-simplifying data submissions. Nucleic Acids Res. 2015;43(Database issue):D1113-6.

60. Kauffmann A, Rayner TF, Parkinson H, Kapushesky M, Lukk M, Brazma A, Huber W. Importing ArrayExpress datasets into R/Bioconductor. Bioinformatics. 2009;25(16):2092-4.

61. Gautier L, Cope L, Bolstad BM, Irizarry RA. Affy-analysis of Affymetrix GeneChip data at the probe level. Bioinformatics. 2004:20(3):307-15.

\section{Submit your next manuscript to BioMed Central and we will help you at every step:}

- We accept pre-submission inquiries

- Our selector tool helps you to find the most relevant journal

- We provide round the clock customer support

- Convenient online submission

- Thorough peer review

- Inclusion in PubMed and all major indexing services

- Maximum visibility for your research

Submit your manuscript at www.biomedcentral.com/submit 